\title{
Expression of Genes Encoding the Enzymes for Glycogen and Trehalose Metabolism in L3 and L4 Larvae of Anisakis simplex
}

\author{
E. Lopieńska-Biernat, E. A. Zaobidna, and M. Dmitryjuk \\ Department of Biochemistry, Faculty of Biology and Biotechnology, University of Warmia and Mazury, Oczapowskiego 1A, \\ 10-917 Olsztyn, Poland \\ Correspondence should be addressed to E. Łopieńska-Biernat; ela.lopienska@uwm.edu.pl
}

Received 2 September 2015; Accepted 22 October 2015

Academic Editor: D. S. Lindsay

Copyright (C) 2015 E. Łopieńska-Biernat et al. This is an open access article distributed under the Creative Commons Attribution License, which permits unrestricted use, distribution, and reproduction in any medium, provided the original work is properly cited.

\begin{abstract}
Trehalose and glycogen metabolism plays an important role in supporting life processes in many nematodes, including Anisakis simplex. Nematodes, cosmopolitan helminths parasitizing sea mammals and humans, cause a disease known as anisakiasis. The aim of this study was to investigate the expression of genes encoding the enzymes involved in the metabolism of trehalose and glycogen-trehalose-6-phosphate synthase (TPS), trehalose-6-phosphate phosphatase (TPP), glycogen synthase (GS), and glycogen phosphorylase (GP) - in stage L3 and stage L4 larvae of A. simplex. The expression of mRNA all four genes, tps, tpp, $g s$, and $g p$, was examined by real-time polymerase chain reaction. The $A$. simplex ribosomal gene (18S) was used as a reference gene. Enzymatic activity was determined. The expression of trehalose enzyme genes was higher in L3 than in L4 larvae, but an inverse relationship was noted for the expression of $g s$ and $g p$ genes.
\end{abstract}

\section{Introduction}

The trehalose synthesis pathway in nematodes proceeds with the participation of trehalose-6-phosphate synthase/ trehalose-6-phosphate phosphatase (TPS/TPP). Biochemical and molecular data for these enzymes are available for several numbers of species. TPS data are available for the following nematodes: Aphelenchus avenae, Ascaris suum, Brugia malayi, Caenorhabditis elegans, Ditylenchus africanus, Heterodera glycines, Heterorhabditis bacteriophora, Onchocerca volvulus, and Panagrolaimus davidi [1-9]. TPP activity has been reported in A. suum, B. malayi, and $C$. elegans [10-12]. Studies investigating TPS and TPP have revealed that TPS activity is a limiting factor in trehalose synthesis because TPP has high affinity for trehalose-6phosphate and hydrolyzes it very quickly to trehalose [13]. Recent studies indicate that, in C. elegans, trehalose increases longevity, prolongs the reproductive period, and contributes to heat tolerance. The inactivation of tps- 1 and tps-2 genes at the cDNA level shortens life expectancy and increases sensitivity to heat stress in C. elegans [14], whereas mutations (knockdown) in the gob-1 gene in the secondary pathway of trehalose synthesis cause intestinal defects in nematodes and contribute to their mortality [10]. The above studies demonstrated that death does not occur in nematodes due to an absence of trehalose and the resulting toxicity of accumulated trehalose-6-phosphate. Those observations provide valuable inputs for research into effective drugs for the treatment of parasitic diseases in humans and animals $[4,11,15]$.

Similar to other organisms, glycogen synthase (GS) in nematodes plays a major role in the synthesis of glycogen, whose properties have been characterized in a limited number of nematode species: A. suum, C. elegans, Litomosoides carinii, and Steinernema feltiae [16-19]. In nematodes, glycogen levels drop during starvation and increase during feeding [20]. GS regulates the level of polysaccharides, which are essential for the survival of parasites [21]. The Sf-gsy-1 gene has been identified in S. feltiae. It has been hypothesized that, during dehydration, trehalose synthesis is intensified at the expense of glycogen synthesis, which is largely controlled by the suppression of the $S f$-gsy-1 gene [19]. Glycogen phosphorylase (GP) is an essential enzyme that regulates the uptake and utilization of glycogen in nematodes [22]. Gp genes have 
been found in draft genome sequences of Trichinella spiralis and Loa loa [23].

Trehalose can be synthesized from glucose, but the interactions between glycogen and trehalose have not been explored to date. There is no evidence that nematodes use glycogen in trehalose synthesis. However, glucose-1phosphate, a product of GP, can be converted to glucose-6phosphate or uridine diphosphoglucose, which are substrates for TPS [13]. Both pathways should be investigated, and the key point for parasite control should be determined to indicate which pathway plays a more significant role for the parasite.

Carbohydrate metabolism plays a vital role in sustaining life processes in many nematodes, including $A$. simplex. The properties of TPS and TPP have been described in stage L3 larvae of A. simplex [24]. The activity of GPa and b has been studied in A. simplex [25], but mRNA levels have not been investigated to date. The synthesis of trehalose and glycogen protects developing parasites against external stressors and enables them to survive in harsh conditions $[19,26]$. The growing incidence of anisakiasis caused by the consumption of raw fish has prompted research into new antiparasitic drugs. The blockade of genes encoding trehalose synthesis enzymes contributes to nematode death, and this observation can be used to develop new antiparasitic treatments [2, 10, 11]. Environmental stressors also reduce the expression of the GS gene, which leads to an increased synthesis of trehalose [19]. The above demonstrates that the synthesis of both saccharides can be controlled at the molecular level in nematodes. The aim of this study was to investigate and compare the expression of genes encoding the enzymes involved in trehalose synthesis (TPS and TPP), glycogen synthesis (GS), and breakdown (GP) in L3 and L4 larvae of A. simplex. This study also tests the hypothesis formulated in our previous work [25], namely, that changes in host environments accompanied by metabolic adaptations associated with changes in enzyme activities probably also induce changes at the genetic level.

\section{Materials and Methods}

2.1. Parasite Material. L3 larvae of A. simplex were collected from fresh Baltic herring (Clupea harengus). The larvae were axenized in antibiotic solution [27] and rinsed several times in sterile saline solution $(0.65 \%)$. L3 larvae were the starting material for the in vitro cultures of L4 larvae of A. simplex, developed according to the method proposed by Iglesias et al. [28]. The larvae were placed in sterile containers, frozen in liquid nitrogen, and stored at $-75^{\circ} \mathrm{C}$. Samples, for enzymatic activity, were then homogenized in an electric homogenizer at a $1: 1(\mathrm{w} / \mathrm{v})$ ratio in TBS buffer. The homogenate was centrifuged at $1500 \mathrm{~g}$ for $15 \mathrm{~min}$ at $4^{\circ} \mathrm{C}$. The supernatant was used to assay for enzymes (TPS, TPP).

2.2. Total RNA Isolation and Reverse Transcription (RT). Total RNA was extracted from both larval stages with the Total RNA Kit (A\&A Biotechnology) according to the manufacturer's protocol. The quantity and purity of isolated RNA were determined by spectrometry using the Nano Drop
1000 spectrophotometer (Thermo Scientific) and by $1.5 \%$ formaldehyde-agarose gel electrophoresis. RT was performed using the reverse transcriptase TranScriba Kit (A\&A Biotechnology, Poland). To eliminate the contamination of genomic DNA, RNA extracts were treated with DNAse/RNAse-free eraser (A\&A Biotechnology), and the mixture was incubated at $37^{\circ} \mathrm{C}$ for $15 \mathrm{~min}$. Total RNA $(2 \mu \mathrm{g})$ was reversetranscribed to cDNA using specific reverse primers (Table 1 ). The $\mathrm{RT}$ reaction was carried out at $37^{\circ} \mathrm{C}$ for $45 \mathrm{~min}$ and at $72^{\circ} \mathrm{C}$ for $10 \mathrm{~min}$. The cDNA product with a final volume of $20 \mu \mathrm{L}$ was stored at $-20^{\circ} \mathrm{C}$ until further analysis. The primers used in the experiment were designed with Primer3 (http://frodo.wi.mit.edu) based on gene sequences in the GenBank database (http://www.ncbi.nlm.nih.gov/). The $A$. simplex ribosomal gene (18S) (U81575) was used as a reference gene.

2.3. Sequencing and Cloning cDNA of tpsl, tpp, gs, and gp from L3 Larvae of A. simplex. Polymerase chain reaction (PCR) was performed with the use of primer sequences shown in Table 1. The reaction mixture consisted of $5 \mu \mathrm{L}$ of cDNA, $25 \mu \mathrm{L}$ of Master Mix Plus High GC (A\&A Biotechnology), $0.1 \mu \mathrm{L}$ of the corresponding forward and reverse primers (Table 1) with the concentration of $100 \mathrm{mM}$, and $23.8 \mu \mathrm{L}$ of nuclease-free water. PCR was performed in a gradient thermocycler (Applied Biosystems) with the following temperature-time profile: $94^{\circ} \mathrm{C}, 3 \mathrm{~min} ;\left(94^{\circ} \mathrm{C}, 30 \mathrm{~s} ; 50^{\circ} \mathrm{C}(58\right.$, $\left.\left.55,53^{\circ} \mathrm{C}\right), 1 \mathrm{~min} ; 72^{\circ} \mathrm{C}, 30 \mathrm{~s}\right) \times 40$ cycles; $72^{\circ} \mathrm{C}, 7 \mathrm{~min}$. PCR products were subjected to electrophoresis on $2 \%$ agarose gel containing $0.01 \%$ ethidium bromide. During cloning into the pJET1 vector $(3.1 \mathrm{~kb}$; Fermentas, Burlington, Ontario, Canada), a nonpurified DNA fragment was reacted with $0.5 \mu \mathrm{L}$ of Walk DNA Polymerase (thermostable polymerase Pwo; A\&A Biotechnology) for $5 \mathrm{~min}$ at $70^{\circ} \mathrm{C}$ in a Gradient thermocycler (Eppendorf). After electrophoresis, the products were isolated from the $2 \%$ gel and purified using the Gel-Out Kit (A\&A Biotechnology). PCR products were thus obtained with blunt ends suitable for cloning into the vector. The ligation reaction was adjusted to $20 \mu \mathrm{L}$. The ligation mixture consisted of $1 \mu \mathrm{L}$ of pJET1 DNA, $9 \mu \mathrm{L}$ of the corresponding tps1, tpp, $g s$, and $g p$ gene fragments, $1 \mu \mathrm{L}$ of DNA T4 ligase (A\&A Biotechnology), and $9 \mu \mathrm{L}$ of water. Ligation was performed for $18 \mathrm{~min}$ at room temperature. The ligation mixture was transformed into competent cells of Escherichia coli TOP10F' (Novagen, Madison, WI, USA). Competent cells were prepared using the E. coli Transformer Kit (A\&A Biotechnology). In the transformation process, $100 \mu \mathrm{L}$ of competent cells was used, to which the entire volume of the ligation mixture was added. The sample was placed on ice for $60 \mathrm{~min}$ and electroporated in Sonic3 (Polsonic, Poland) for $3 \mathrm{~s}$. The prepared samples were distributed on plates with LA (Luria Agar) solid medium with ampicillin $(50 \mu \mathrm{L} / \mathrm{mL})$. The plates were incubated for $16 \mathrm{~h}$ at $37^{\circ} \mathrm{C}$. The potential presence of clones in the colonies was checked for $g s, g p$ and $t p s l$, tpp gene fragments by PCR using primers and reaction conditions. Transformant colonies where the presence of the gene had been confirmed were transferred to $5 \mathrm{~mL}$ of liquid LB (Luria Broth) medium with ampicillin $(50 \mu \mathrm{L} / \mathrm{mL})$ and incubated for $17 \mathrm{~h}$ at $37^{\circ} \mathrm{C}$. Recombinant 
TABLE 1: Nucleotide sequences of primers for PCR amplification of genes encoding the enzymes for glycogen metabolism.

\begin{tabular}{|c|c|c|c|c|}
\hline Primers & Names & Primers $\left(5^{\prime}-3^{\prime}\right)$ & Annealing temperature $\left({ }^{\circ} \mathrm{C}\right)$ & Size of PCR product (bp) \\
\hline For. & tps1s & GGGTCTTGGGAGATCAACA & \multirow{2}{*}{50} & \multirow{2}{*}{268} \\
\hline Rev. & tpslas & TGCTGCTTTGGTGTCAACTC & & \\
\hline For. & tpps & AGCGTTATTCAGTGGCTCGT & \multirow{2}{*}{58} & \multirow{2}{*}{192} \\
\hline Rev. & tppas & CATGGCACTCTTTGTTGGTG & & \\
\hline For. & gsFor & CAAYGTBGARTCVCTSAAAGGHC & \multirow{2}{*}{55} & \multirow{2}{*}{485} \\
\hline Rev. & gsRev & ATRAARCADCCRAADCCDGAVAG & & \\
\hline For. & gps & GATCGCCGTAAGCAGATCAGCGT & \multirow{2}{*}{53} & \multirow{2}{*}{927} \\
\hline Rev. & gpas & CGGCATCCATACTTGCTTGATTTG & & \\
\hline
\end{tabular}

TABLE 2: Primer sequences used for semiquantitative and real-time PCR.

\begin{tabular}{lccc}
\hline Gene & Accession number & Primers $\left(5^{\prime}-3^{\prime}\right)$ & Size of PCR product $(\mathrm{bp})$ \\
\hline$t p s$ & $* /$ KJ560557 & $\begin{array}{r}\text { For. GAAGTTACGTCAATCAATATGAGAAGG/ACTACGCATCACAAGCAACG } \\
\text { Rev. TTCAGGTCCACCCACCCATC/CGCTTGACGTATCAATGGAA }\end{array}$ & 163 \\
\hline$t p p$ & $* /$ KJ560558 & $\begin{array}{r}\text { For. GTTTTCGCTTGTCTGCTCACG/TACGAAAGCATTCCAACGTG } \\
\text { Rev. TCGTTAGCGGCATTTCCTG/ATCGGATGATACGCTGCAAG }\end{array}$ & 193 \\
\hline$g s$ & JX173686 & $\begin{array}{l}\text { For. GCCACCGATTTGCACACACAA } \\
\text { Rev. CAGCCACGCAAATTCCTCA }\end{array}$ & 173 \\
\hline$g p$ & JX941465 & $\begin{array}{l}\text { For. GGATTCGCACACAACAATACTA } \\
\text { Rev. RCTGCGTCTTCTCGATCTCTTG }\end{array}$ & 211 \\
\hline $18 S$ & U81575 & For. ACCAGTAACGAAAGCGTGTG & 2193 \\
\hline
\end{tabular}

${ }^{*}$ This sequence data was used to design primers for real-time PCR.

plasmids were isolated from cultures using the Plasmid Mini AX Kit (A\&A Biotechnology). The presence of gene fragments in the resulting plasmids was investigated by PCR based on the above protocol. PCR product sequencing was performed in the 3730xI DNA Analyzer (Applied Biosystems, Carlsbad, CA, USA).

2.4. Sequence Analysis of Partial tps1, tpp, gs and $g p m R N A$ in L3 Larvae of A. simplex. Analyses of tpsl, tpp, gs, and $g p$ and sequence alignment were performed in Geneious 7.0 created by Biomatters (available from http://www.geneious.com/).

2.5. Relative Quantification of Gene Expression. Relative quantification of gene expression by real-time PCR was performed to compare tps1, tpp, $g s$, and $g p$ transcription levels in L3 and L4 larvae of A. simplex. Fold changes in target genes, normalized to $18 \mathrm{~S}$ and relative to expression levels in the larvae, were calculated using the comparative Ct $\left(2^{-\Delta \Delta C t}\right)$ method [29]. Quantitative real-time PCR was performed using SYBRGreen Taq PCR-MIX (A\&A Biotechnology, Poland) according to the manufacturer's protocol. A quantity of $20 \mu \mathrm{L}$ of the reaction solution contained $1 \mu \mathrm{L}$ of the template (1:10 dilution of cDNA product), $10 \mu \mathrm{L}$ of SYBRGreen Taq PCR-MIX (2x), $1 \mu \mathrm{L}$ of $10 \mu \mathrm{M}$ of each primer (Table 2), $6.6 \mu \mathrm{L}$ of water, and $0.4 \mu \mathrm{L}$ of ROX Reference dye II. The following thermal cycling conditions were applied: $10 \mathrm{~min}$ at $95^{\circ} \mathrm{C}$ followed by 38 cycles of $20 \mathrm{~s}$ at $95^{\circ} \mathrm{C}, 20 \mathrm{~s}$ at $55^{\circ} \mathrm{C}$, and $30 \mathrm{~s}$ at $72^{\circ} \mathrm{C}$. The data were analyzed and normalized relative to transcript levels of $18 \mathrm{~S}$ ribosomal
RNA genes in the AB analytical application (7500v2.0). All samples were tested in triplicate in the FAST7500 LightCycler (Applied Biosystems, USA). Melting curves were developed after amplification.

2.6. TPS and TPP Activity. TPS activity was determined based on the method described by Giaever et al. [30] and TPP activity was marked on the method proposed by Kaasen et al. [31]. Trehalose, the end-product of these reactions, was determined by high-performance liquid chromatography according to the procedure described by Dmitryjuk et al. [7]. The activity of TPS and TPP was expressed in units (U) per mg of protein measured spectrophotometrically at $280 \mathrm{~nm}$ in the Nano Drop ND-1000 spectrophotometer (Thermo Fisher Scientific, Waltham, MA, USA). One unit defines the quantity of synthesized trehalose $(\mu \mathrm{mol})$ during $1 \mathrm{~min}$ at $37^{\circ} \mathrm{C}$. The results are presented as mean values from five independent measurements with five replicates per sample.

2.7. Statistical Analysis. Data are expressed as mean values and standard deviations. The statistical significance of differences between samples at both developmental stages was analyzed by one-way analysis of variance (ANOVA) and Tukey's test in the SPSS 16.0 application at a significance level of $P<0.05$. Pearson's correlation test was used for determination of the correlation between the enzymes activities of TPS, TPP, GS, and GP and the expression of $t p s 1$, tpp, $g s$, and $g p$ mRNA in L4 larvae of A. simplex. 


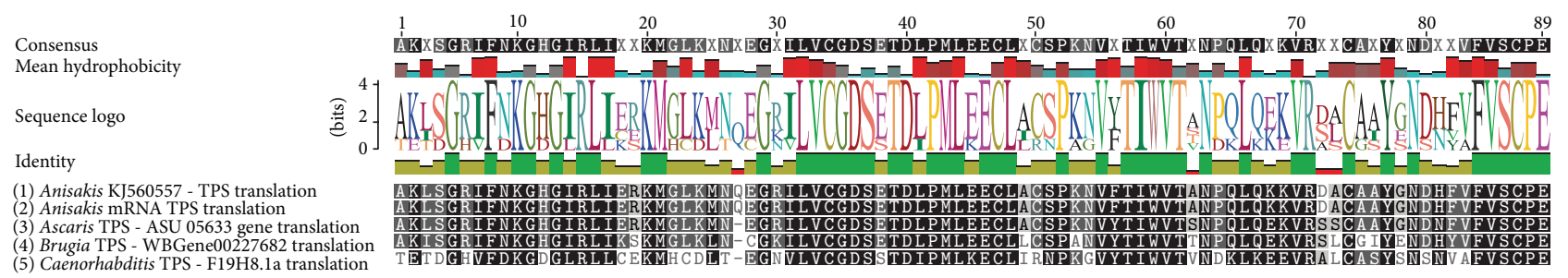

(a)

Consensus

Mean hydrophobicity

Sequence logo

Identity

(1) Anisakis TPP - KJ560558 translation

(2) Anisakis TPP - mRNA translation

(3) Ascaris TPP - ASU_05137 translation

(4) Brugia TPP - Bm4641 translation

(5) Caenorhabditis TPP - H13N06.3a translation

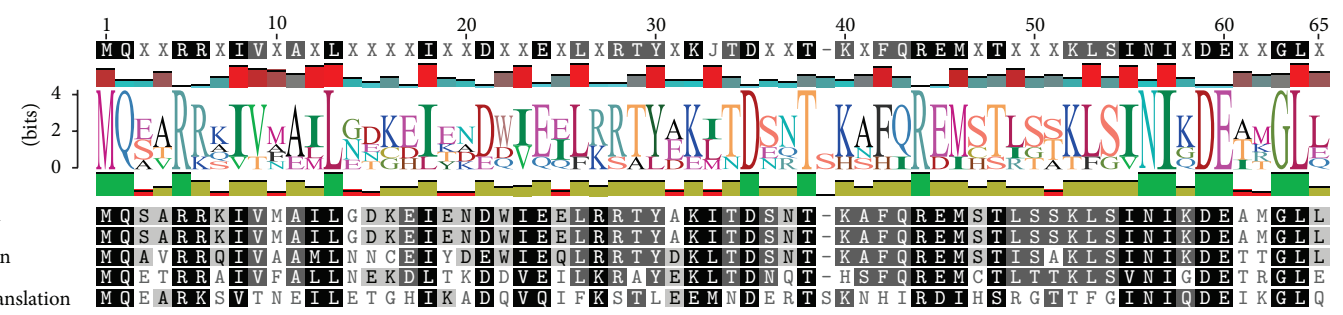

(b)

Figure 1: Multiple alignment of predicted partial deduced amino acid sequences of trehalose-6-phosphate synthase (a) and trehalose-6phosphate phosphatase (b) from nematodes: third-stage (L3) larvae of Anisakis simplex, Caenorhabditis elegans, Brugia malayi, and Ascaris suum. The first line shows the consensus sequence of numbers amino acid position. Below (mean hydrophobicity) is a histogram of the hydrophobicity of individual amino acids. The line sequence logo is a consensus sequence where the size of the symbol of the amino acid determines its stability in its use position (this value also describes a line identity), and each amino acid is indicated by another color. Tinting the background of individual items in sequence on alignment determines similarity in position on all sequences.

\section{Results}

3.1. Sequencing of Trehalose-6-Phosphate Synthase and Phosphatase Genes. Gene fragments encoding parts of TPS and TPP in L3 larvae of $A$. simplex were isolated and sequenced. The fragments encoding sequences for tps and tpp had a length of $268 \mathrm{bp}$ and $192 \mathrm{bp}$, respectively (Figures 1(a) and (b)). These sequences of mRNA have not been deposited in GenBank, being too short.

3.2. Sequencing of Glycogen Synthase and Phosphorylase Genes. The genes encoding GS and GP in L3 larvae of $A$. simplex were isolated in part and sequenced. The fragments encoding sequences for $g s$ and $g p$ had a length of $485 \mathrm{bp}$ and $927 \mathrm{bp}$, respectively. The analyzed fragments were deposited in GenBank (accessions numbers JX173686 and JX941465.1). Predicted translated products were 162 amino acids for AFP95337.1 and 309 amino acids for AFX58973.1 (Figures 2(a) and $2(\mathrm{~b}))$.

3.3. Analysis of A. simplex Gene Expression by Real-Time PCR. Relative quantification of the expression of four genes in the L3 and L4 larval stages was performed by real-time RTPCR using cDNA from the total RNA of L3 and L4 larvae of $A$. simplex. The analysis confirmed differences in gene expression between L3 and L4 larvae that had been observed using semiquantitative PCR. The expression patterns were grouped into two profiles: (1) the $t p s 1$ gene was characterized by a 3 -fold increase and the tpp gene by a 5 -fold increase in expression in L3 larvae relative to L4 larvae, and the differences between developmental stages were significant; (2) the expression of the $g s$ gene was 3-fold higher and the expression of the $g p$ gene was 1.2-fold higher in L4 than in L3 stages (Figure 3).
3.4. Activity of A. simplex Trehalose Synthesis Enzymes. The activity of both trehalose synthesis enzymes was higher in L3 than in L4 larvae. The activity of TPS increased 2-fold, but the activity of TPP was higher by 10 -fold in L3 than in older larvae. The differences in the activity levels of both enzymes in L3 and L4 larvae were statistically significant $(P<0.05)$ (Figure 3).

The correlation between the activity of enzymes for trehalose and glycogen metabolism and their mRNA expression is shown in Figure 3. The correlation coefficient was higher $(r=0.9, P<0.005)$ between trehalose synthesis enzymes and mRNA expression. Meanwhile, a moderate correlation was also obtained between enzymes of glycogen metabolism and mRNA expression values $(r=-0.8, P<0.005)$ (Figure 3 ).

\section{Discussion}

The A. simplex genome has been partially sequenced. Scaffolds of genomic DNA have been submitted to GenBank (http://www.ncbi.nlm.nih.gov/), but they do not reveal active genes. An analysis of expressed sequence tags in L3 larvae of A. simplex constitutes an important part of research. EST revealed that $20 \%$ of matched clones were highly homologous with the genes or proteins of C. elegans [32]. The above achievement and the completely sequenced C. elegans [33] facilitated and contributed to the identification of genes in A. simplex. Molecular research based on known homologous sequences in C. elegans did not produce positive results, and partial success was achieved only when degraded primers, designed based on $A$. suum and B. malayi sequences, were used.

Trehalose is a disaccharide that protects various organisms against environmental stressors such as dehydration, 


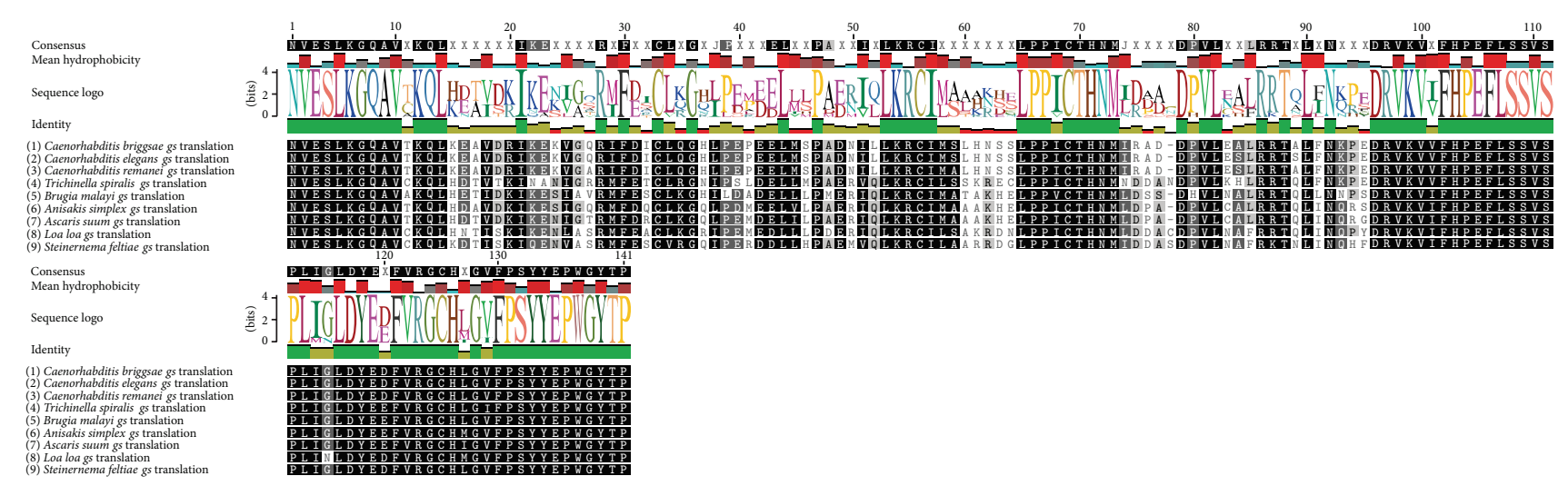

(a)
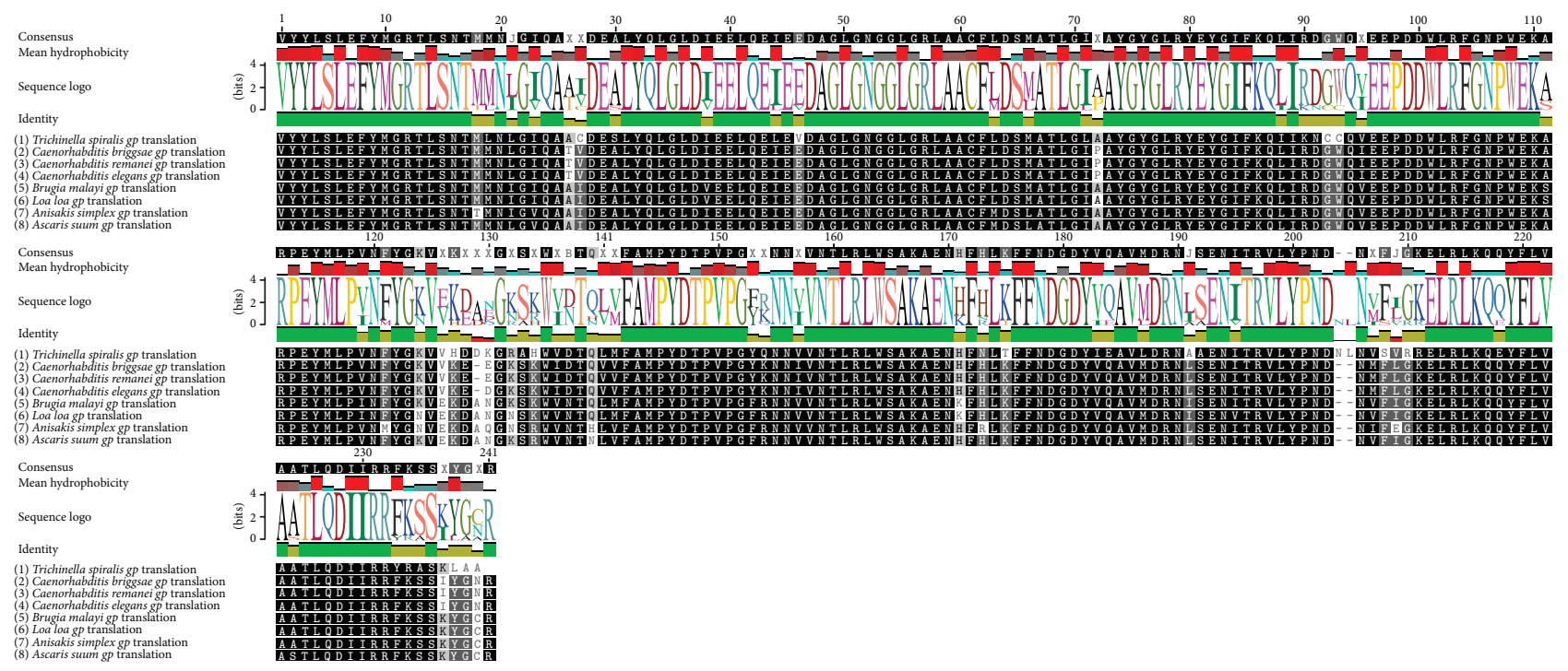

(b)

Figure 2: Multiple alignment of predicted partial deduced amino acid sequences of glycogen synthase (a) and glycogen phosphorylase (b) from nematodes: third-stage (L3) larvae of Anisakis simplex, Loa loa, Caenorhabditis elegans, C. remanei, C. briggsae, Trichinella spiralis, Brugia malayi, Ascaris suum, and Steinernema feltiae. The legend is the same as in Figure 1.

anoxia, or changes in temperature [14]. L3 larvae, which have as their natural host heterothermic organisms such as fish and cephalopods, are extremely viable and resistant. However, the fourth larval stage (L4) develops in the alimentary tract of the definitive host, that is, marine mammals, or in humans [3436]. In recent studies, C. elegans was used to explore trehalose metabolism $[2,14]$. Those studies investigated the expression and function of the gene encoding the TPS enzyme, which participates in trehalose synthesis in C. elegans and other nematode species. Two TPS encoding genes were identified in C. elegans: tps-1 (4351 bp) and tps-2 (3862 bp), and the products of both genes were classified into the glycosyltransferase 20 family $[2,37]$. Two tps genes with very high resemblance to the tps-2 gene of $C$. elegans were also identified in $A$. avenae, but the expression of a gene similar to $C e$-tps- 1 has not yet been confirmed. Aav-TPS-2 and Ce-TPS-2 proteins exhibit $62 \%$ similarity, whereas Aav-TPS-1 and Ce-TPS-1 are characterized by $49 \%$ similarity, and the sequences of both genes in A. avenae and C. elegans show $51 \%$ similarity. The two genes encoding TPS are often not expressed in multicellular animals [9]. In this study, the tps-1 gene in the L3 larvae of A. simplex was partially identified and its expression was demonstrated at the cDNA level, but the presence of the tps-2 gene was not confirmed. The presence of a one gene encoding the TPS enzyme and only partial cDNA may be difficult to identify in $A$. simplex larvae because not all genes are active in the analyzed developmental stage and mRNA is very unstable. Our bioinformatics analysis of genomic DNA confirmed the presence of a single form of the gene, TPS1 (GenBank accession number KJ560557). The expression of the tps gene has not been explored to date. The partial tps gene in L3 larvae of $A$. simplex has a length of $268 \mathrm{bp}$. The resulting gene of $A$. simplex invasive larvae is less similar to tps genes in other parasites, including O. volvulus (421 bp) and B. malayi (834 bp) [2]. The translated sequenced gene in L3 larvae of $A$. simplex shows $58.5 \%$ similarity to the Ce-tps-1 gene (Figure 1(a)). The second stage of trehalose synthesis catalyzes the TPP enzyme. The enzyme has been 


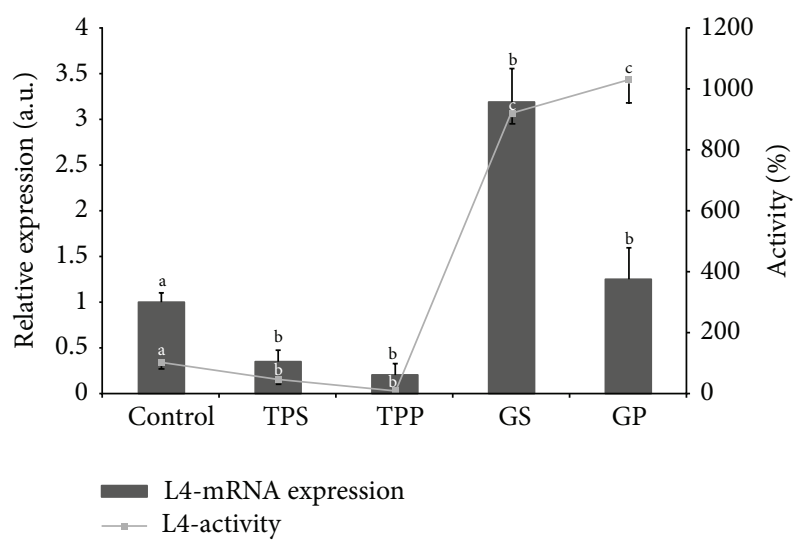

FIgURE 3: Comparison of relative activity of enzymes and expression of four genes encoding the enzymes trehalose-6-phosphate synthase (TPS), trehalose-6-phosphate phosphatase (TPP), glycogen synthase (GS), and glycogen phosphorylase (GP) using the normalization reference gene $r y b$ between L3 and L4 larval stages of Anisakis simplex. Different letters ( $\mathrm{a}, \mathrm{b}$, and $\mathrm{c}$ ) above the bars represent significant differences between L3 (control) and L4 of A. simplex for gene expression and enzyme activity. $¥$ Data from Łopieńska-Biernat et al. [25], $r=0.95$ for enzymes and mRNA of trehalose metabolism, $r=-0.8$ for glycogen metabolism in L4 of $A$. simplex $(P<0.005)$ (as control $100 \%$ activity of enzymes was $0.43 \pm$ $0.02 \mathrm{U} / \mathrm{mg}$ for TPS and $0.34 \pm 0.03$ for TPP U/mg in L3).

studied at the genetic level in two organisms: C. elegans and B. malayi where one gene ( $g o b-1, t p p)$ encoding the TPP enzyme was identified $[10,11]$. Expression of the partial tpp gene in L3 larvae of A. simplex was determined in this study, and sequence homology with genomic DNA was confirmed in 98\% (GenBank accession number KJ560557). The similarity between the sequence of the translated tpp gene in A. suum and other tpp genes, which were used to design the primers, was only $37.4 \%$, and the similarity between the analyzed t $p p$ gene and the gob-1 gene was only $21.1 \%[4,38]$.

Glycogen occurs commonly in living organisms as a major source of energy [39]. In invertebrates, glycogen is a source of energy during intensified metabolic processes [40]. The glycogen synthesis pathway is highly conserved in eukaryotes, and it involves the same enzymes in different organisms, from yeasts to humans [41]. The expression of GS genes has also been studied in the limited organisms: $C$. elegans, where the activity of $g s y-1$ was observed [42], S. feltiae (Sf-gsy-1) [19], and B. malayi [43]. The analyses conducted in this study with the involvement of specific primers supported the identification of $g s$ expression at the cDNA level in L3 larvae of $A$. simplex. The partially sequenced $g s$ gene in $A$. simplex has a length of $485 \mathrm{bp}$ and is shorter than $g s y-1$ in $C$. elegans (2427 bp) and B. malayi (512 bp), but longer than in S. feltiae (327 bp) and Loa loa (327 bp). The gene identified in A. simplex exhibits high similarity to other GS genes in different species, including $72 \%$ similarity to the $g s y-1$ gene in C. elegans and $73 \%$ similarity in B. malayi (Figure 2(a)). Changes in the expression of the $S f-g s y$-1 gene were noted in dehydrated and rehydrated $S$. feltiae parasites, which could indicate that glycogen synthesis protects nematodes against dehydration. Increased trehalose synthesis in response to desiccation stress takes place at the expense of glycogen synthesis, which is largely controlled by the suppression of the $S f-g s y-1$ gene [19]. It can be assumed that similar changes occur in L3 larvae of $A$. simplex in response to environmental stress. The analyses performed in this study with the use of specific primers supported the determination of $g p$ expression at the cDNA level in L3 larvae of $A$. simplex. The partial sequence of the $g p$ gene in $A$. simplex has a length of $927 \mathrm{bp}$ and is shorter than the $g p$ gene in C. elegans (2000 bp), L. loa (LOAG0616) (2565 bp), and Trichinella spiralis (2844 bp) [22]. The cluster analysis of nematodes revealed that $g p$ and $g s$ products in A. simplex exhibit high sequence similarity to analogous mRNA in parasitic nematodes ( $L$. loa and B. malayi) and free-living nematodes (C. elegans) (Figure 2(b)).

The expression of trehalose and glycogen metabolism genes is difficult to discuss due to the absence of published data concerning parasitic nematodes. The activity of trehalose synthesis enzymes is correlated with the expression of genes encoding those enzymes. The activity of trehalose enzymes and their genes was higher in L3 than in L4 larvae of $A$. simplex. The demonstrated high positive correlation also confirms the crucial role of trehalose metabolism in L3 larvae of A. simplex (Figure 3). Sex-based differences in the expression of trehalose and glycogen genes were also observed in C. elegans. Male nematodes, which are smaller and more sensitive to stress, exhibit a higher expression of trehalose genes than hermaphrodites, such as young larvae of A. simplex [44].

The expression levels of genes responsible for glycogen breakdown and synthesis transcripts were higher in older larvae (Figure 3). An inverse relationship was noted between the predominance of GS transcripts over GP transcripts in A. simplex and the predominance of GP over GS in dauer larvae of C. elegans [42]. Similar results were reported in a previous study investigating the activity of glycogen enzymes in L4 larval stage [25], which revealed higher activity levels of GP than GS in older larvae of $A$. simplex (Figure 3).

Our results suggest that L3 larvae produce trehalose rather than glycogen, whereas the opposite could be true in L4 larvae of $A$. simplex. The above hypothesis was formulated in view of the higher expression of trehalose synthesis genes and lower expression of glycogen synthesis genes in L3 larvae compared to in L4 larvae of A. simplex (Figure 3). Our results and fungus Magnaporthe oryzae [45] suggest that probably glycogen breakdown $(g p)$ is a significant factor in regulation genes expression as source glucose and substrate for synthesis trehalose or synthesis trehalose $(t p p)$ as toxicity of accumulated T6P $[10,15]$. The parasite can probably be controlled in the metabolic pathways of both sugars, trehalose and glycogen.

Trehalose and glycogen metabolism plays a vital role in most living organisms $[14,39]$. The expression of tpsl, tpp, and $g s$ and $g p$ genes in A. simplex points to the importance of trehalose and glycogen metabolism in the life processes of parasites and expands our knowledge of nematode genomes. 


\section{Conflict of Interests}

The authors declare that there is no conflict of interests regarding the publication of this paper.

\section{Acknowledgment}

The authors would like to thank Ph.D. Student $Ł$. Paukszto, from the Department of Plant Physiology, Genetics and Biotechnology, University Warmia and Mazury, for bioinformatic analyses.

\section{References}

[1] S. H. Loomis, K. A. C. Madin, and J. H. Crowe, "Anhydrobiosis in nematodes: biosynthesis of trehalose," Journal of Experimental Zoology, vol. 211, no. 3, pp. 311-320, 1980.

[2] F. I. Pellerone, S. K. Archer, C. A. Behm, W. N. Grant, M. J. Lacey, and A. C. Somerville, "Trehalose metabolism genes in Caenorhabditis elegans and filarial nematodes," International Journal for Parasitology, vol. 33, no. 11, pp. 1195-1206, 2003.

[3] D. A. Wharton, "The environmental physiology of Antarctic terrestrial nematodes: a review," Journal of Comparative Physiology $B$, vol. 173, no. 8, pp. 621-628, 2003.

[4] K. Goyal, J. A. Browne, A. M. Burnell, and A. Tunnacliffe, "Dehydration-induced tps gene transcripts from an anhydrobiotic nematode contain novel spliced leaders and encode atypical GT-20 family proteins," Biochimie, vol. 87, no. 6, pp. 565-574, 2005.

[5] G. B. Jagdale, P. S. Grewal, and S. O. Salminen, "Both heatshock and cold-shock influence trehalose metabolism in an entomopathogenic nematode," Journal of Parasitology, vol. 91, no. 5, pp. 988-994, 2005.

[6] N. W. Alkharouf, V. P. Klink, I. B. Chouikha et al., "Timecourse microarray analyses reveal global changes in gene expression of susceptible Glycine max (soybean) roots during infection by Heterodera glycines (soybean cyst nematode)," Planta, vol. 224, no. 4, pp. 838-852, 2006.

[7] M. Dmitryjuk, E. Łopieńska-Biernat, and M. Farjan, “The level of sugars and synthesis of trehalose in Ascaris suum tissues," Journal of Helminthology, vol. 83, no. 3, pp. 237-243, 2009.

[8] M. Dmitryjuk, M. Dopieralska, E. Łopieńska-Biernat, and R. J. Fraczek, "Trehalose phosphate synthase from muscles of adult Ascaris suum-purification and partial characterization," Journal of Helminthology, vol. 87, pp. 212-221, 2013.

[9] A. Haegeman, J. Jacob, B. Vanholme, T. Kyndt, M. Mitreva, and G. Gheysen, "Expressed sequence tags of the peanut pod nematode Ditylenchus africanus: the first transcriptome analysis of an Anguinid nematode," Molecular and Biochemical Parasitology, vol. 167, no. 1, pp. 32-40, 2009.

[10] J. D. Kormish and J. D. McGhee, "The C. elegans lethal gutobstructed gob-1 gene is trehalose-6-phosphate phosphatase," Developmental Biology, vol. 287, no. 1, pp. 35-47, 2005.

[11] S. Kushwaha, P. K. Singh, A. K. Rana, and S. MisraBhattacharya, "Cloning, expression, purification and kinetics of trehalose-6-phosphate phosphatase of filarial parasite Brugia malayi," Acta Tropica, vol. 119, no. 2-3, pp. 151-159, 2011.

[12] M. Dmitryjuk, E. Łopieńska-Biernat, and B. Sawczuk, "Properties of trehalose-6-phosphate phosphatase from Ascaris suum muscles-preliminary studies," Russian Journal of Nematology, vol. 20, no. 1, pp. 9-14, 2012.
[13] C. A. Behm, "The role of trehalose in the physiology of nematodes," International Journal for Parasitology, vol. 27, no. 2, pp. 215-229, 1997.

[14] Y. Honda, M. Tanaka, and S. Honda, "Trehalose extends longevity in the nematode Caenorhabditis elegans," Aging Cell, vol. 9, no. 4, pp. 558-569, 2010.

[15] J. D. Farelli, B. D. Galvin, Z. Li et al., "Structure of the trehalose-6-phosphate phosphatase from Brugia malayi reveals key design principles for anthelmintic drugs," PLoS Pathogens, vol. 10, no. 7, Article ID e1004245, 2014.

[16] K. A. Madin and J. H. Crowe, "Anhydrobiosis in nematodes: carbohydrate and lipid metabolism during dehydration," Journal of Experimental Zoology, vol. 193, no. 3, pp. 335-342, 1975.

[17] P. R. Komuniecki and H. J. Saz, "The effect of levamisole on glycogen synthase and the metabolism of Litomosoides carinii," Journal of Parasitology, vol. 68, no. 2, pp. 221-227, 1982.

[18] L. L. Hannigan, M. J. Donahue, and R. A. Masaracchia, "Comparative purification and characterization of invertebrate muscle glycogen synthase from the porcine parasite Ascaris suum," The Journal of Biological Chemistry, vol. 260, no. 30, pp. 16099-16105, 1985.

[19] T. Z. Gal, A. Solomon, I. Glazer, and H. Koltai, "Alterations in the levels of glycogen and glycogen synthase transcripts during desiccation in the insect-killing nematode Steinernema feltiae IS-6," Journal of Parasitology, vol. 87, no. 4, pp. 725-732, 2001.

[20] M. J. Donahue, N. J. Yacoub, M. R. Kaeini, and B. G. Harris, "Activity of enzymes regulating glycogen metabolism in perfused muscle-cuticle sections of Ascaris suum (Nematoda)," Journal of Parasitology, vol. 67, no. 3, pp. 362-367, 1981.

[21] R. A. Masaracchia, M. R. Rantala, and M. J. Donahue, "Glycogenin-dependent organization of Ascaris suum muscle glycogen," Journal of Parasitology, vol. 86, no. 6, pp. 1206-1212, 2000.

[22] K. Zółtowska, E. Łopieńska-Biernat, and J. Rokicki, “Activity of glycogen catabolism enzymes in Contracaecum rudolphii (Anisakidae)," Russian Journal of Nematology, vol. 17, no. 1, pp. 11-16, 2009.

[23] M. Mitreva, D. P. Jasmer, D. S. Zarlenga et al., "The draft genome of the parasitic nematode Trichinella spiralis," Nature Genetics, vol. 43, no. 3, pp. 228-235, 2011.

[24] E. Łopieńska-Biernat, M. Czubak, E. A. Zaobidna, and J. Rokicki, "Properties of trehalose-6-phosphate synthase and trehalose-6-phosphate phosphatase in third-stage larvae of the nematode Anisakis simplex-preliminary studies," Russian Journal of Nematology, vol. 22, part 2, pp. 131-140, 2014.

[25] E. Łopieńska-Biernat, K. Zółtowska, and J. Rokicki, "Glycogen catabolism enzymes and protein fractions in the third and fourth larval stages of Anisakis simplex," Journal of Helminthology, vol. 82, no. 1, pp. 45-51, 2008.

[26] D. A. Wharton, G. Goodall, and C. J. Marshall, "Freezing survival and cryoprotective dehydration as cold tolerance mechanisms in the Antarctic nematode Panagrolaimus davidi," Journal of Experimental Biology, vol. 206, no. 2, pp. 215-221, 2003.

[27] L. Iglesias, A. Valero, and F.-J. Adroher, "Some factors which influence the in vitro maintenance of Anisakis simplex (Nematoda)," Folia Parasitologica, vol. 44, no. 4, pp. 297-301, 1997.

[28] L. Iglesias, A. Valero, R. Benítez, and F. J. Adroher, "In vitro cultivation of Anisakis simplex: pepsin increases survival and moulting from fourth larval to adult stage," Parasitology, vol. 123, no. 3, pp. 285-291, 2001. 
[29] M. W. Pfaffl, "A new mathematical model for relative quantification in real-time RT-PCR," Nucleic Acids Research, vol. 29, no. 9, article e45, 2001.

[30] H. M. Giaever, O. B. Styrvold, I. Kaasen, and A. R. Strøm, "Biochemical and genetic characterization of osmoregulatory trehalose synthesis in Escherichia coli," Journal of Bacteriology, vol. 170, no. 6, pp. 2841-2849, 1988.

[31] I. Kaasen, J. McDougall, and A. R. Strøm, "Analysis of the otsBA operon for osmoregulatory trehalose synthesis in Escherichia coli and homology of the OtsA and OtsB proteins to the yeast trehalose-6-phosphate synthase/phosphatase complex," Gene, vol. 145, no. 1, pp. 9-15, 1994.

[32] H. S. Yu, S. K. Park, K. H. Lee et al., "Anisakis simplex: analysis of expressed sequence tags (ESTs) of third-stage larva," Experimental Parasitology, vol. 117, no. 1, pp. 51-56, 2007.

[33] The C. elegans Sequencing Consortium, "Genome sequence of the nematode C. elegans: a platform for investigating biology," Science, vol. 282, no. 5396, pp. 2012-2018, 1998.

[34] A. M. Adams, K. S. Miller, M. M. Wekell, and F. M. Dong, "Survival of Anisakis simplex in microwave-processed arrowtooth flounder (Atheresthes stomias)," Journal of Food Protection, vol. 62, no. 4, pp. 403-409, 1999.

[35] M. T. Audicana, I. J. Ansotegui, L. F. De Corres, and M. W. Kennedy, "Anisakis simplex: dangerous-dead and alive?" Trends in Parasitology, vol. 18, no. 1, pp. 20-25, 2002.

[36] A. D. Molina-García and P. D. Sanz, "Anisakis simplex larva killed by high-hydrostatic-pressure processing," Journal of Food Protection, vol. 65, no. 2, pp. 383-388, 2002.

[37] P. M. Coutinho, E. Deleury, G. J. Davies, and B. Henrissat, "An evolving hierarchical family classification for glycosyltransferases," Journal of Molecular Biology, vol. 328, no. 2, pp. 307-317, 2003.

[38] A. R. Jex, S. Liu, B. Li et al., “Ascaris suum draft genome," Nature Research Letter, vol. 479, pp. 529-535, 2011.

[39] P. J. Roach, A. V. Skurat, and R. A. Harris, "Regulation of glycogen metabolism," in Comprehensive Physiology, vol. 7, pp. 609-647, Wiley Online Library, 2001.

[40] T. N. Palmer and P. A. Fournier, "Replenishment of muscle glycogen after high-intensity exercise: a role for intramuscular lactate glyconeogenesis?" Biochemical Society Transactions, vol. 25, no. 1, pp. 25-30, 1997.

[41] B. A. Pederson, C. Cheng, W. A. Wilson, and P. J. Roach, "Regulation of glycogen synthase," Journal of Biological Chemistry, vol. 275, no. 36, pp. 27753-27761, 2000.

[42] S. J. Holt and D. L. Riddle, "SAGE surveys C. elegans carbohydrate metabolism: evidence for an anaerobic shift in the longlived dauer larva," Mechanisms of Ageing and Development, vol. 124, no. 7, pp. 779-800, 2003.

[43] E. Ghedin, S. Wang, D. Spiro, and et al, "Draft genome of the filarial nematode parasite Brugia malayi," Science, vol. 317, no. 5845, pp. 1756-1760, 2007.

[44] C. Miersch and F. Döring, "Sex differences in carbohydrate metabolism are linked to gene expression in Caenorhabditis elegans," PLoS ONE, vol. 7, no. 9, Article ID e44748, 18 pages, 2012.

[45] M. Badaruddin, L. J. Holcombe, R. A. Wilson, Z.-Y. Wang, M. J. Kershaw, and N. J. Talbot, "Glycogen metabolic genes are involved in trehalose-6-phosphate synthase-mediated regulation of pathogenicity by the rice blast fungus Magnaporthe oryzae," PLoS Pathogens, vol. 9, no. 10, Article ID e1003604, 2013. 

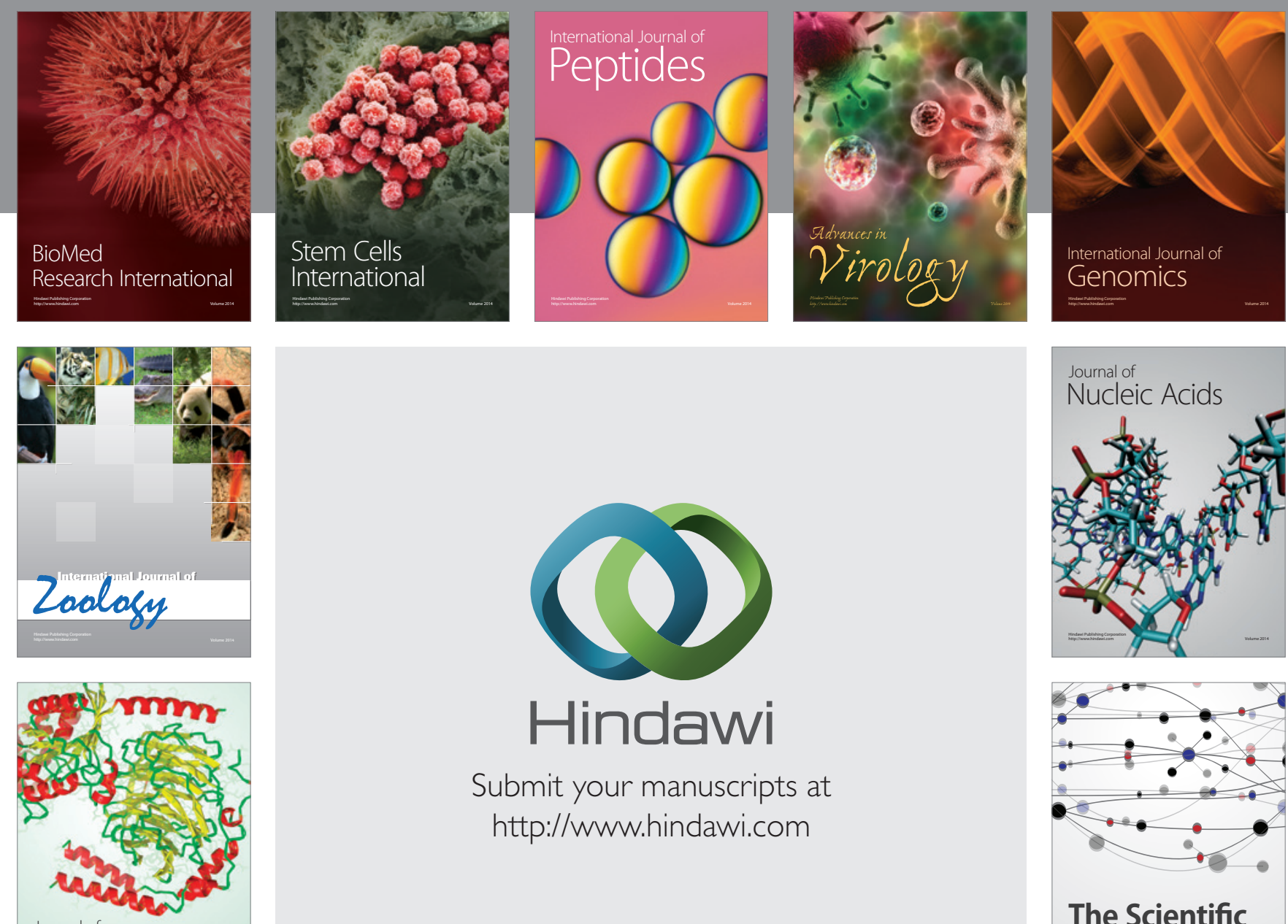

Submit your manuscripts at

http://www.hindawi.com

Journal of
Signal Transduction
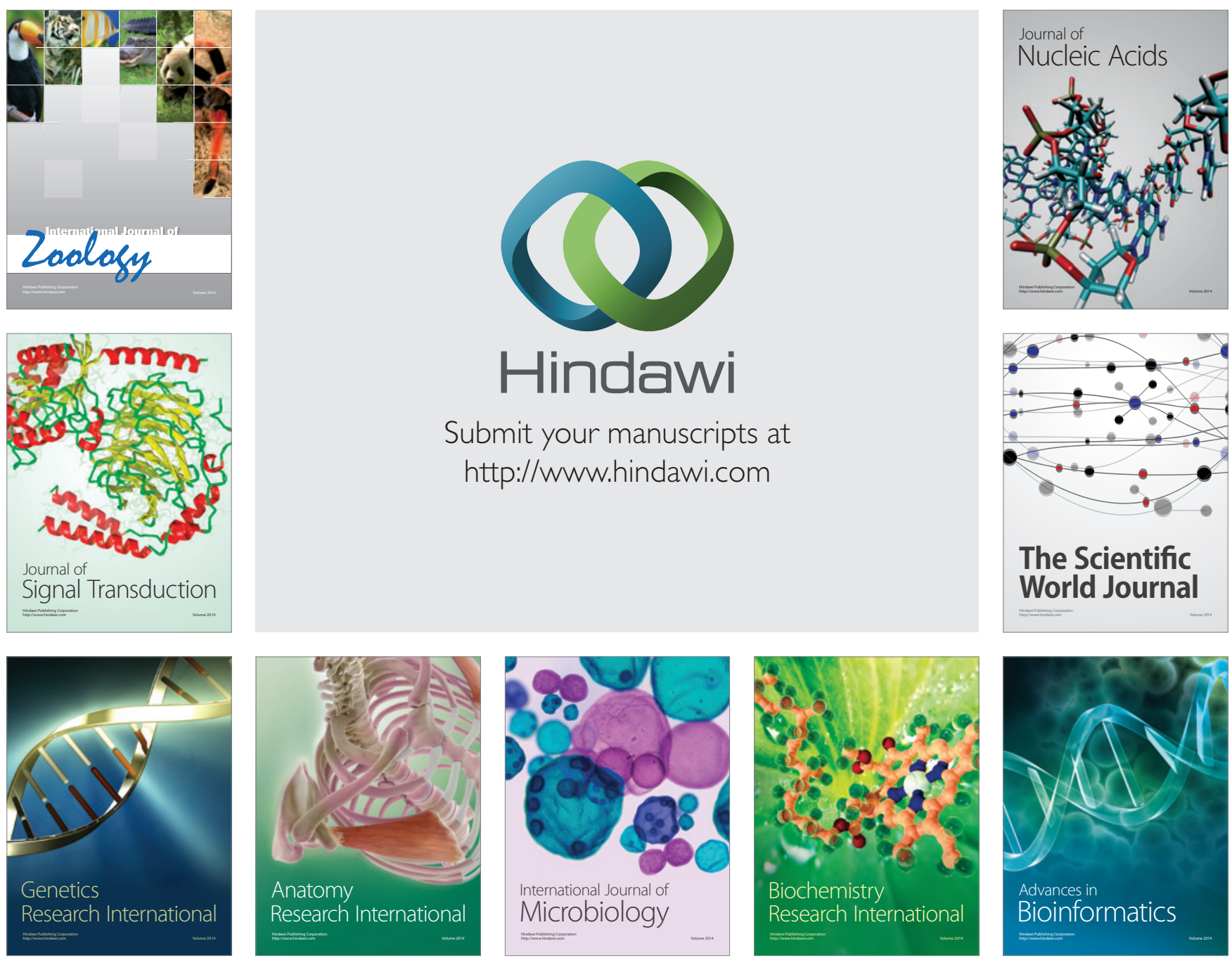

The Scientific World Journal
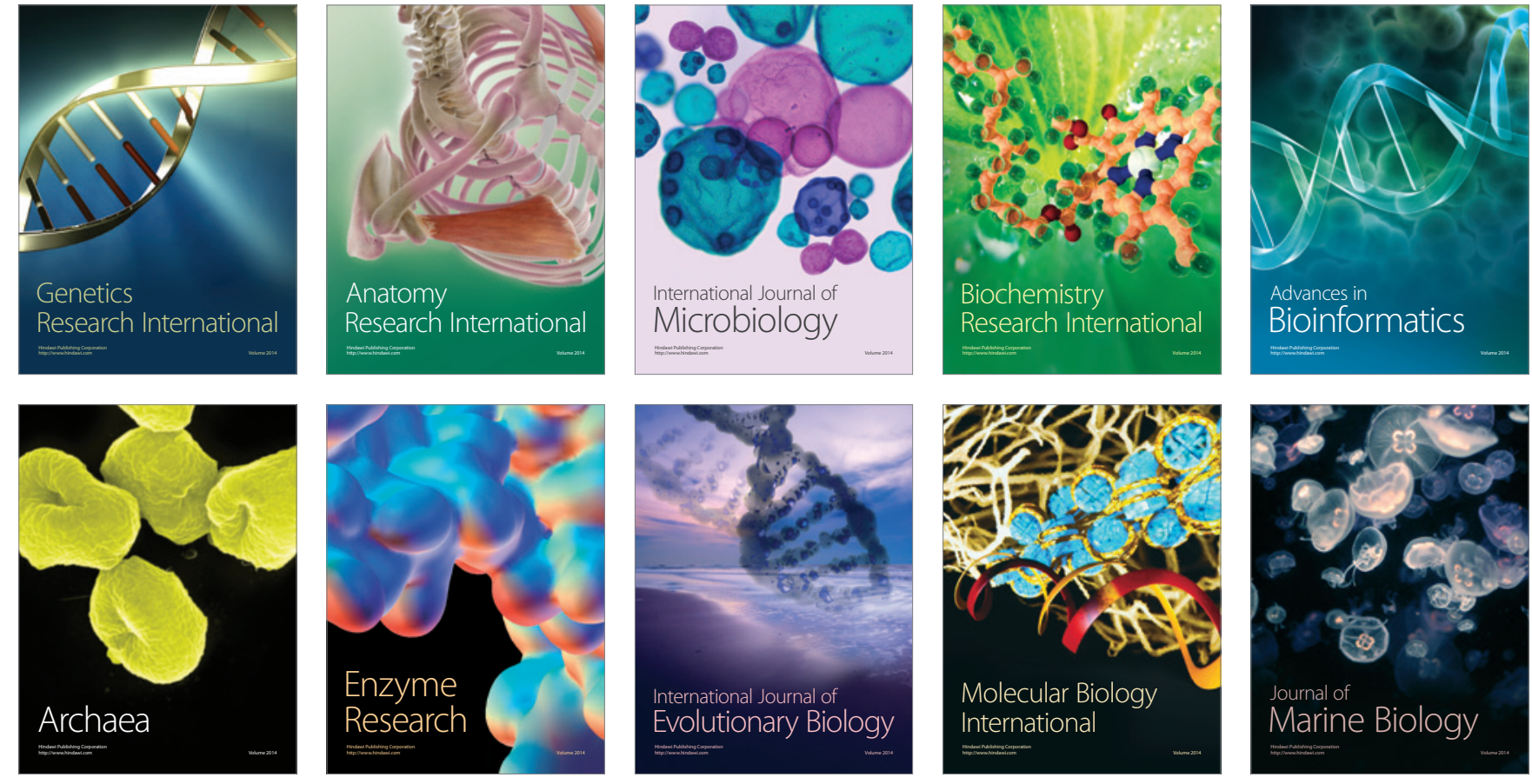